\title{
Hospitalization before surgery increases risk for postoperative infections
}

\author{
Marta Kelava, MD, ${ }^{a}$ Michael Robich, MD, MSPH, ${ }^{b}$ Penny L. Houghtaling, MS, ${ }^{\mathrm{c}}$ Joseph F. Sabik III, MD, ${ }^{\mathrm{b}}$ \\ Steven Gordon, MD, ${ }^{\mathrm{d}}$ Tomislav Mihaljevic, MD, ${ }^{\mathrm{b}}$ Eugene H. Blackstone, MD, ${ }^{\mathrm{b}, \mathrm{c}}$ and \\ Colleen G. Koch, MD, MS, MBA ${ }^{\mathrm{a}}$
}

\begin{abstract}
Objectives: Exposure to a health care facility before surgery may increase risk for postoperative infections. Our objectives were to (1) determine whether the prevalence of postoperative infections was higher among patients who were hospitalized before cardiac surgery, (2) identify risk factors for infection, and (3) evaluate in-hospital outcomes.
\end{abstract}

\begin{abstract}
Methods: A total of 32,707 patients underwent cardiac surgery from January 1, 2000, to January 1, 2011. Forty percent $(13,107)$ were hospitalized before their surgery date or were transfers from other health care facilities, and $60 \%(19,600)$ were same-day admissions. The primary outcome consisted of a composite infection: pneumonia, sepsis, surgical site infection, and urinary tract infection. The secondary outcome was in-hospital death. The propensity method was used to compare infectious complications and mortality between groups.
\end{abstract}

Results: Overall infectious complications occurred in 2327 patients (7.1\%). Overall composite and individual infections decreased over the study period $(P$ for trend $<.0001)$. Among 7814 propensity-matched pairs, 522 infections $(6.7 \%)$ occurred in the same-day admission group versus $676(8.7 \%)$ in the prior hospitalization group, $P<.0001$. In-hospital mortality was $1.5 \%(\mathrm{n}=120)$ for the same-day admission group versus $2.8 \%$ $(\mathrm{n}=221)$ for the prior hospitalization group $(P<.0001)$.

Conclusions: Although the risk of infection decreased over time, the relationship between exposure to a health care facility before surgical intervention and higher infection risk remained substantial. Further investigation into processes of care surrounding infection control is necessary to reduce postoperative infections and associated morbidity. (J Thorac Cardiovasc Surg 2014;148:1615-21)

Supplemental material is available online.

Postoperative nosocomial infections are among the most common noncardiac complications, with an estimated prevalence of $2.7 \%$ to $21.7 \% .^{1-3}$ Although several patientspecific factors are reported to be associated with infection

\footnotetext{
From the Departments of Cardiothoracic Anesthesia ${ }^{a}$ and Thoracic and Cardiovascular Surgery, ${ }^{\mathrm{b}}$ Heart and Vascular Institute, Department of Quantitative Health Sciences, ${ }^{c}$ Research Institute, and Department of Infectious Disease, ${ }^{\mathrm{d}}$ Medicine Institute, Cleveland Clinic, Cleveland, Ohio.

This study was funded in part by the Cleveland Clinic Department of Thoracic and Cardiovascular Surgery, the Sheikh Hamdan bin Rashid Al Maktoum Distinguished Chair in Thoracic and Cardiovascular Surgery (held by Dr Sabik), and the Kenneth Gee and Paula Shaw, PhD, Chair in Heart Research (held by Dr Blackstone).

Disclosures: Authors have nothing to disclose with regard to commercial support.

Received for publication March 25, 2014; revisions received June 2, 2014; accepted for publication June 5, 2014.

Address for reprints: Colleen G. Koch, MD, MS, MBA, Cleveland Clinic, Department of Cardiothoracic Anesthesia, 9500 Euclid Ave, J4-331, Cleveland, OH 44195 (E-mail: kochc@ccf.org).

$0022-5223 / \$ 36.00$

Copyright (C) 2014 by The American Association for Thoracic Surgery

http://dx.doi.org/10.1016/j.jtcvs.2014.06.067
}

risk, evidence suggests that exposure to a health care facility before surgery increases infectious complications postoperatively. ${ }^{4-7}$ Patients who acquire infections postoperatively have higher morbidity and mortality, and use of hospital resources is increased. ${ }^{1,3,4,8}$ Because patients cannot be randomized to hospitalization before surgery, this investigation is considered a natural experiment. ${ }^{9}$ To propose methods of ameliorating infection risk in cardiac surgery, the objectives of this study were to (1) determine whether the occurrence of postoperative infections was higher among patients who were hospitalized before surgery, (2) identify risk factors for infection, and (3) evaluate inhospital mortality after infection.

\section{PATIENTS AND METHODS \\ Patients}

From January 1, 2000, to January 1, 2011, 32,707 adult patients underwent isolated coronary artery bypass grafting (CABG), an isolated valve procedure, combined $\mathrm{CABG}$ and valve surgery (with or without addition of a maze procedure or atrial septal defect repair), or ascending aorta replacement at Cleveland Clinic. A total of 13,107 patients $(40 \%)$ were hospitalized before the surgery date or were transferred from other health care facilities; 19,600 (60\%) presented for surgery on the day of operation. Patients who underwent heart or lung transplantation, major left ventricular procedures, endarterectomies, 


\section{Abbreviations and Acronyms \\ $\mathrm{CABG}=$ coronary artery bypass grafting \\ $\mathrm{SD}=$ standard deviation}

septal myectomies, or other procedures were excluded from this investigation. Perioperative variables were retrieved from the Cardiovascular Information Registry, a prospective database approved for use in research by the Institutional Review Board, with individual patient consent waived.

\section{End Points}

Our primary end point was a composite of postoperative infectious complications: surgical incision site infections (sternal wound, thoracotomy incision, leg and arm harvest site), septicemia, pneumonia, and urinary tract infections. A secondary end point was all-cause mortality evaluated with respect to prior hospitalization status.

\section{Statistical Analyses}

Categorical data are summarized by frequencies and percentages and continuous data by mean \pm standard deviation (SD), or 15 th, 50th (median), and 85th percentiles (equivalent to $\pm 1 \mathrm{SD}$ ) when the distribution was skewed. Comparisons were made using the $\chi^{2}$ test, Fisher's exact test, or the Wilcoxon rank-sum test where appropriate. Uncertainty is expressed by confidence limits equivalent to \pm 1 standard error $(68 \%)$.

\section{Propensity-Matched Comparison}

The characteristics of the patients with prior hospitalization differed substantially from those of patients admitted on the day of surgery (Table 1). Therefore, the propensity-score method was used to obtain 2 cohorts of comparable patients for comparison of hospital outcomes. ${ }^{10-12}$ Multivariable logistic regression analysis was used to identify the patient characteristics most associated with prior hospitalization (parsimonious model). The variables considered in this analysis are listed in Appendix E1. Variable selection, with a $P$-value criterion for retention of variables in the model of .05 , used bagging (bootstrap aggregation), ${ }^{13,14}$ with automated analysis of 500 resampled data sets, followed by tabulation of the frequency of occurrence of both single factors and closely related clusters of factors. Factors with occurrence of $50 \%$ or greater were retained in the final model. Thereafter, the parsimonious model was augmented with other variables representing patient demography, symptoms, cardiac and noncardiac comorbidities, and procedure variables (semisaturated propensity model). From this, a propensity score was calculated for each patient by solving the saturated model for the probability of being in the prior hospitalization group.

Using only the propensity score, previously hospitalized patients were matched to same-day surgery patients using a greedy matching strategy. ${ }^{15}$ Patients whose propensity scores deviated more than 0.15 were considered unmatched. Greedy matching based on propensity scores yielded 7814 well-matched pairs (Figures 1 and 2 and Table 1).

\section{Risk Factors for Infectious Complications}

The occurrence of infection, time to infection, and temporal trends in the occurrence of postoperative infections were assessed. Multivariable logistic regression was performed to identify factors associated with postoperative infectious complications using the variables listed in Appendix E1 and bagging as described in the previous section.

All analyses were performed using SAS statistical software version 9.2 (SAS, Inc, Cary, NC).

\section{RESULTS \\ Postoperative Infections}

Prevalence. There were 2327 postoperative infectious complications overall $(7.1 \%)$ among the 32,707 patients. Among the 7814 propensity-matched pairs, $676(8.7 \%)$ experienced composite infection in the prior hospitalization group versus $522(6.7 \%)$ in the same-day admission group, $P<.0001$. The difference was largely related to a higher prevalence of septicemia, pneumonia, and urinary tract infections in the prior hospitalization group (Table 2).

Prevalence among unmatched patients. Unmatched patients who were hospitalized before surgery had more comorbidities and a more unstable clinical presentation compared with unmatched same-day admission patients. These unmatched patients with prior hospitalizations had a higher prevalence of postoperative infections, 14\% compared with $3.2 \%$ for unmatched same-day admission patients, $P<.0001$ (Table E1).

Timing. Among matched patients already hospitalized at the time of surgery, the median time from surgery to postoperative infection was 7.6 days (15th and 85th percentiles, 2.6 and 16 days, respectively; range, 0-74 days). Among matched patients admitted on the same day, the respective duration was 7.5 days (15th and 85 th percentiles, 2.7 and 15 days, respectively; range, 0-53 days).

Temporal trends. The overall number of same-day admissions and hospital transfers remained relatively unchanged over the study period (Figure 3). A temporal trend of decreased prevalence of all types of infections was observed $(P<.0001$; Figures 4 and 5$)$.

Risk factors. Several demographic, comorbidity, clinical presentation, and procedural factors were associated with a higher risk of composite infection (Table 3). Hospitalization before surgery was associated with a 1.9-fold increase in infection risk, greater than that of other comorbidities, including diabetes. Emergency surgery and intraoperative red blood cell transfusion were the only other variables that placed a patient at greater or similar risk of infection as prior hospitalization.

\section{Mortality}

Unadjusted in-hospital mortality was $0.85 \%(n=167)$ for the same-day admission groups and 4.0\% $(\mathrm{n}=522)$ for the prior hospitalization group $(P<.0001)$; for matched patients, it was $1.5 \%(\mathrm{n}=120)$ and $2.8 \%(\mathrm{n}=221)$, respectively $(P<.0001)$. Mortality was lower among unmatched same-day admission patients $(0.4 \%)$ than patients hospitalized before surgery $(5.7 \% ; P<.0001)$ (Table E1).

\section{DISCUSSION}

\section{Principal Findings}

Hospitalization before cardiac surgery was associated with more postoperative infectious complications than 
TABLE 1. Patient and operative characteristics by same-day and prior hospital admission status: before and after propensity matching

\begin{tabular}{|c|c|c|c|c|c|c|c|c|c|}
\hline \multirow[b]{3}{*}{ Variable } & \multicolumn{4}{|c|}{ Same-day admission } & \multirow[b]{3}{*}{$\boldsymbol{P} \dagger$} & \multicolumn{4}{|c|}{ Prior admission } \\
\hline & \multicolumn{2}{|c|}{$\begin{array}{l}\text { Before matching } \\
(\mathrm{n}=\mathbf{1 9 , 6 0 0})\end{array}$} & \multicolumn{2}{|c|}{$\begin{array}{l}\text { After matching } \\
\quad(\mathbf{n}=\mathbf{7 8 1 4})\end{array}$} & & \multicolumn{2}{|c|}{$\begin{array}{l}\text { After matching } \\
(\mathrm{n}=\mathbf{7 8 1 4})\end{array}$} & \multicolumn{2}{|c|}{$\begin{array}{l}\text { Before matching } \\
(\mathrm{n}=\mathbf{1 3 , 1 0 7 )}\end{array}$} \\
\hline & $\mathbf{n}^{*}$ & $\begin{array}{l}\text { No. }(\%) \text { or } \\
\text { mean } \pm \text { SD }\end{array}$ & $\mathbf{n}^{*}$ & $\begin{array}{l}\text { No. }(\%) \text { or } \\
\text { mean } \pm \text { SD }\end{array}$ & & $\mathbf{n}^{*}$ & $\begin{array}{l}\text { No. }(\%) \text { or } \\
\text { mean } \pm \text { SD }\end{array}$ & $\mathbf{n}^{*}$ & $\begin{array}{l}\text { No. }(\%) \text { or } \\
\text { mean } \pm \text { SD }\end{array}$ \\
\hline \multicolumn{10}{|l|}{ Demographics } \\
\hline Age (y) & 19,600 & $63 \pm 14$ & 7814 & $66 \pm 13$ & .10 & 7814 & $66 \pm 13$ & 13,107 & $66 \pm 13$ \\
\hline Female & 19,600 & $5831(30)$ & 7814 & $2543(33)$ & .3 & 7814 & $2606(33)$ & 13,107 & $4869(37)$ \\
\hline Race & 19,464 & & 7741 & & & 7703 & & 12,916 & \\
\hline White & & $17,669(91)$ & & $6860(89)$ & .6 & & $6804(88)$ & & $11,206(87)$ \\
\hline Black & & $618(3.2)$ & & $417(5.4)$ & .3 & & $445(5.8)$ & & $1101(8.5)$ \\
\hline Other & & $1177(6.0)$ & & $464(6.0)$ & .8 & & $454(5.9)$ & & $609(4.7)$ \\
\hline Body surface area $\left(\mathrm{m}^{2}\right)$ & 19,594 & $2.0 \pm 0.26$ & 7810 & $2.0 \pm 0.27$ & .5 & 7809 & $2.0 \pm 0.28$ & 13,097 & $2.0 \pm 0.28$ \\
\hline Hospital transfer & 19,600 & $0(0)$ & 7814 & $0(0)$ & - & 7771 & $3189(41)$ & 13,030 & $6122(47)$ \\
\hline \multicolumn{10}{|l|}{ Clinical presentation } \\
\hline NYHA functional class & 19,593 & & 7810 & & .004 & 7812 & & 13,102 & \\
\hline I & & $5725(29)$ & & $1427(18)$ & & & $1426(18)$ & & $1943(15)$ \\
\hline II & & $10,113(52)$ & & $4037(52)$ & & & $3874(50)$ & & $5767(44)$ \\
\hline III & & $3364(17)$ & & $2002(26)$ & & & $2088(27)$ & & $3925(30)$ \\
\hline IV & & $391(2.0)$ & & $344(4.4)$ & & & $424(5.4)$ & & $1467(11)$ \\
\hline Emergency surgery & 19,597 & $78(0.40)$ & 7814 & $76(0.97)$ & .0002 & 7814 & $128(1.6)$ & 13,107 & $486(3.7)$ \\
\hline \multicolumn{10}{|l|}{ Cardiac comorbidity } \\
\hline LVEF (\%) & 15,857 & $53 \pm 11$ & 6370 & $49 \pm 12$ & .6 & 6505 & $49 \pm 12$ & 10,866 & $47 \pm 13$ \\
\hline Myocardial infarction & 19,600 & $4476(23)$ & 7814 & $3006(38)$ & .3 & 7814 & $3072(39)$ & 13,107 & $6117(47)$ \\
\hline Heart failure & 19,600 & $3271(17)$ & 7814 & $2338(30)$ & .003 & 7813 & $2508(32)$ & 13,106 & $5793(44)$ \\
\hline Endocarditis & 19,600 & $612(3.1)$ & 7814 & $284(3.6)$ & .05 & 7814 & $331(4.2)$ & 13,107 & 997 (7.6) \\
\hline Atrial fibrillation/flutter & 17,395 & $1530(8.8)$ & 6794 & 809 (12) & .6 & 7534 & $873(12)$ & 12,667 & $1600(13)$ \\
\hline Prior cardiac surgery & 19,600 & $3770(19)$ & 7814 & $2119(27)$ & .2 & 7814 & $2188(28)$ & 13,107 & $3942(30)$ \\
\hline No. of cardiac operations & 19,600 & & 7814 & & .4 & 7814 & & 13,107 & \\
\hline 1 & & $15,830(81)$ & & $5695(73)$ & & & $5626(72)$ & & $9165(70)$ \\
\hline 2 & & $3175(16)$ & & $1688(22)$ & & & $1712(22)$ & & $2961(23)$ \\
\hline$\geq 3$ & & $595(3.0)$ & & $431(5.5)$ & & & $476(6.1)$ & & $981(7.5)$ \\
\hline Complete heart block or pacer & 17,395 & $680(3.9)$ & 6794 & $403(5.9)$ & .5 & 7534 & $427(5.7)$ & 12,667 & $792(6.3)$ \\
\hline Ventricular arrhythmia & 17,395 & $1602(9.2)$ & 6794 & $633(9.3)$ & .6 & 7534 & $722(9.6)$ & 12,667 & $1215(9.6)$ \\
\hline \multicolumn{10}{|l|}{ Noncardiac comorbidity } \\
\hline Carotid disease & 19,600 & $4906(25)$ & 7814 & $3008(38)$ & .6 & 7814 & 3037 (39) & 13,107 & $5746(44)$ \\
\hline Hypertension & 19,600 & $12,583(64)$ & 7814 & $6004(77)$ & .8 & 7814 & $5994(77)$ & 13,107 & $10,356(79)$ \\
\hline Pharmacologically treated diabetes & 19,444 & $3259(17)$ & 7720 & $2019(26)$ & .5 & 7697 & $2049(27)$ & 12,856 & $3832(30)$ \\
\hline Insulin-treated diabetes & 19,422 & $1142(5.9)$ & 7710 & $779(10)$ & .2 & 7687 & $821(11)$ & 12,836 & $1675(13)$ \\
\hline PAD & 19,600 & $1460(7.4)$ & 7814 & $996(13)$ & .9 & 7814 & $1003(13)$ & 13,107 & $2007(15)$ \\
\hline COPD & 19,600 & $2361(12)$ & 7814 & $1369(18)$ & .5 & 7813 & $1403(18)$ & 13,106 & $2776(21)$ \\
\hline Smoking & 19,600 & $9897(51)$ & 7760 & $4593(59)$ & .7 & 7763 & $4574(59)$ & 13,018 & $8047(62)$ \\
\hline Renal dialysis & 19,600 & $91(0.46)$ & 7814 & $75(0.96)$ & .0007 & 7813 & $122(1.6)$ & 13,106 & $376(2.9)$ \\
\hline Stroke & 19,600 & $1056(5.4)$ & 7814 & $746(9.5)$ & .15 & 7814 & $800(10)$ & 13,107 & $1860(14)$ \\
\hline Creatinine (mg/dL) & 19,555 & $1.07 \pm 0.62$ & 7795 & $1.16 \pm 0.85$ & .04 & 7811 & $1.18 \pm 0.84$ & 13,099 & $1.29 \pm 1.07$ \\
\hline Bilirubin (g/dL) & 19,496 & $0.68 \pm 0.41$ & 7751 & $0.67 \pm 0.45$ & .02 & 7417 & $0.69 \pm 0.47$ & 12,458 & $0.71 \pm 0.59$ \\
\hline Glomerular filtration rate (MDRD) & 19,555 & $78 \pm 22$ & 7795 & $74 \pm 25$ & .04 & 7811 & $74 \pm 26$ & 13,099 & $71 \pm 29$ \\
\hline Hematocrit (\%) & 19,593 & $40 \pm 5.3$ & 7808 & $38 \pm 5.6$ & .002 & 7814 & $38 \pm 5.8$ & 13,105 & $36 \pm 6.2$ \\
\hline \multicolumn{10}{|l|}{ Procedure } \\
\hline Isolated CABG & 19,600 & $4379(22)$ & 7814 & $2847(36)$ & $<.0001$ & 7814 & $3127(40)$ & 13,107 & $5160(39)$ \\
\hline Isolated valve & 19,600 & $8588(4)$ & 7814 & $1910(24)$ & .7 & 7814 & $1932(25)$ & 13,107 & $2964(23)$ \\
\hline Combined CABG/valve & 19,600 & $3472(18)$ & 7814 & $1742(22)$ & $<.0001$ & 7814 & $1472(19)$ & 13,107 & $2752(21)$ \\
\hline Aortic root, ascending aorta & 19,600 & $2615(13)$ & 7814 & $1138(15)$ & .6 & 7814 & $1113(14)$ & 13,107 & $1903(15)$ \\
\hline Other cardiac & 19,600 & $546(2.8)$ & 7814 & $177(2.3)$ & .7 & 7814 & $170(2.2)$ & 13,107 & $328(2.5)$ \\
\hline
\end{tabular}




\begin{tabular}{|c|c|c|c|c|c|c|c|c|c|}
\hline \multirow[b]{3}{*}{ Variable } & \multicolumn{4}{|c|}{ Same-day admission } & \multirow[b]{3}{*}{$\boldsymbol{P} \dagger$} & \multicolumn{4}{|c|}{ Prior admission } \\
\hline & \multicolumn{2}{|c|}{$\begin{array}{l}\text { Before matching } \\
\quad(\mathrm{n}=19,600)\end{array}$} & \multicolumn{2}{|c|}{$\begin{array}{l}\text { After matching } \\
\quad(\mathrm{n}=7814)\end{array}$} & & \multicolumn{2}{|c|}{$\begin{array}{l}\text { After matching } \\
\quad(\mathrm{n}=\mathbf{7 8 1 4})\end{array}$} & \multicolumn{2}{|c|}{$\begin{array}{l}\text { Before matching } \\
\quad(\mathrm{n}=13,107)\end{array}$} \\
\hline & n* & $\begin{array}{l}\text { No. }(\%) \text { or } \\
\text { mean } \pm \text { SD }\end{array}$ & n* & $\begin{array}{l}\text { No. }(\%) \text { or } \\
\text { mean } \pm \text { SD }\end{array}$ & & n* & $\begin{array}{l}\text { No. }(\%) \text { or } \\
\text { mean } \pm \text { SD }\end{array}$ & n* & $\begin{array}{l}\text { No. }(\%) \text { or } \\
\text { mean } \pm \text { SD }\end{array}$ \\
\hline Surgical invasiveness & 19,598 & & 7812 & & & 7813 & & 13,106 & \\
\hline Full incision & & $14,453(74)$ & & $7302(93)$ & .7 & & $7289(93)$ & & $12,493(95)$ \\
\hline Less invasive & & $4691(24)$ & & $46(6)$ & .3 & & $43(5.6)$ & & $503(3.8)$ \\
\hline Percutaneous/ports & & $454(2.3)$ & & $42(0.54)$ & $<.0001$ & & $88(1.1)$ & & $110(0.84)$ \\
\hline $\mathrm{CPB}$ & 19,600 & $18,776(96)$ & 7814 & $7308(94)$ & .07 & 7814 & $7250(93)$ & 13,107 & $12,123(92)$ \\
\hline CPB time (min) & 19,600 & $93 \pm 43$ & 7814 & $99 \pm 48$ & .008 & 7814 & $101 \pm 50$ & 13,107 & $106 \pm 54$ \\
\hline Myocardial ischemic time (min) & 19,600 & $72 \pm 34$ & 7814 & $76 \pm 37$ & .04 & 7814 & $77 \pm 39$ & 13,107 & $79 \pm 41$ \\
\hline Requirement for IABP & 19,600 & $205(1.0)$ & 7814 & $142(1.8)$ & .8 & 7814 & $146(1.9)$ & 13,107 & $387(3.0)$ \\
\hline \multicolumn{10}{|l|}{ Blood product use } \\
\hline $\mathrm{RBC}$ transfusion & 19,593 & 7347 (37) & 7809 & $4209(54)$ & $<.0001$ & 7807 & 4603 (59) & 13,095 & $8920(68)$ \\
\hline FFP & 19,591 & $1801(9.2)$ & 7807 & $1112(14)$ & $<.0001$ & 7805 & $1492(19)$ & 13,091 & 3089 (24) \\
\hline Any blood product & 19,600 & $7973(41)$ & 7814 & $4472(57)$ & $<.0001$ & 7814 & $4854(62)$ & 13,107 & $9262(71)$ \\
\hline Immunosuppressive therapy & 19,600 & $2740(14)$ & 7814 & $1120(14)$ & .6 & 7814 & $1142(15)$ & 13,107 & $2111(16)$ \\
\hline \multicolumn{10}{|l|}{ Outcome } \\
\hline In-hospital death & 19,600 & $167(0.85)$ & 7814 & $120(1.5)$ & $<.0001$ & 7814 & $221(2.8)$ & 13,107 & $522(4.0)$ \\
\hline
\end{tabular}

$S D$, Standard deviation; NYHA, New York Heart Association; $L V E F$, left ventricular ejection fraction; $P A D$, peripheral arterial disease; $C O P D$, chronic obstructive pulmonary disease; $M D R D$, Modification of Diet in Renal Disease formula; $C A B G$, coronary artery bypass grafting; $C P B$, cardiopulmonary bypass; $I A B P$, intra-aortic balloon pump; $R B C$, red blood cell; FFP, fresh frozen plasma. *Patients with data available. †Matched group comparison.

same-day admission, even after accounting for commonly reported risk factors for infection in this setting. Most infections occurred beyond a week postoperatively, but at our institution, their frequency is decreasing.

Prevalence. An investigation from the 1980s reported that longer length of hospital stay before cardiac surgery

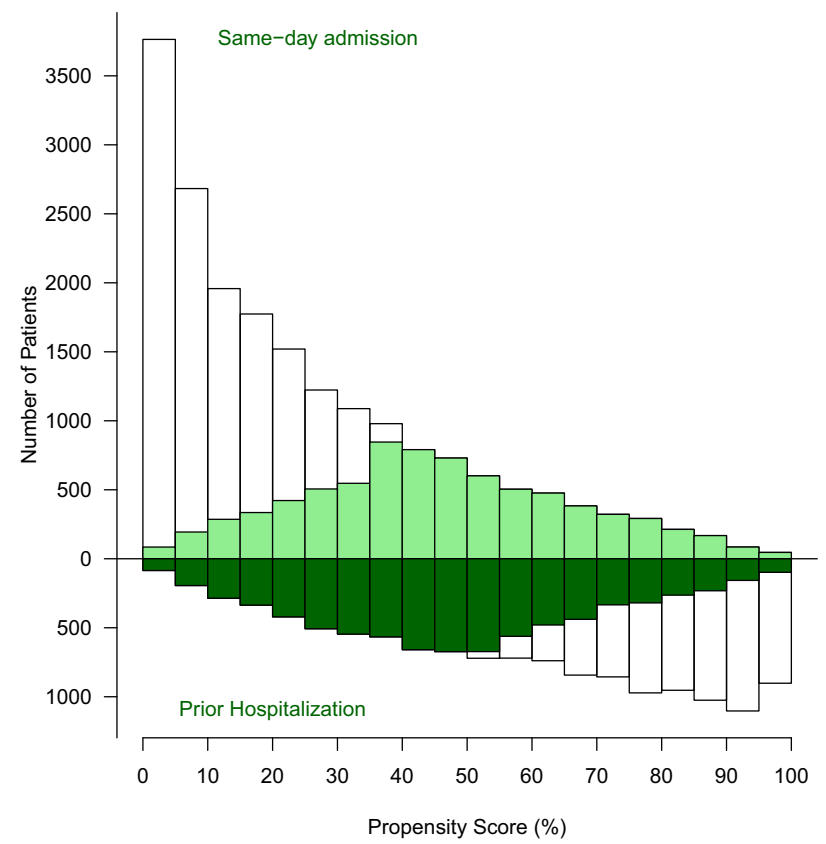

FIGURE 1. Mirrored histogram of propensity scores for patients who were hospitalized before surgery and those who were same-day admissions. Shaded areas represent propensity-matched groups. increased infectious risk postoperatively. Although the investigators noted that increased risk could reflect the severity of illness, they also believed it was partially attributable to exposure to the hospital's microorganisms. ${ }^{7}$ An investigation from a noncardiac surgical setting using the American College of Surgeons National Safety Quality Improvement Project found increased risk for surgical site infections, pneumonia, and urinary tract infections in patients who were hospitalized preoperatively (odds ratio, 1.21; confidence interval, 1.06-1.39). They also attributed colonization with bacteria from the health care setting and the presence of foreign catheters as potential risk factors. ${ }^{6}$ Other investigators have also reported on increased infectious risks associated with hospitalization before surgical procedures; however, few comment on practical preventive measures. ${ }^{5,6}$ Although inpatient hospitalization may be considered a surrogate for greater acuity of illness, it also remained a risk factor after propensity matching accounted for high-risk comorbid conditions, complex procedures, and emergency surgery. Occurrence of postoperative infections is related to the presence of devices (urinary catheters, central venous cannulation, and arterial line monitoring), extensive surgical incision sites, and routine need for postoperative ventilatory support. ${ }^{3,6,16}$ Timing. Using the Nationwide In-patient Sample, Vogel and colleagues ${ }^{4}$ reported that hospitalization before CABG was associated with increased postoperative infections (pneumonia, urinary tract infections, and sepsis) in an incremental manner, with increasing days of preoperative hospitalization associated with more postoperative 


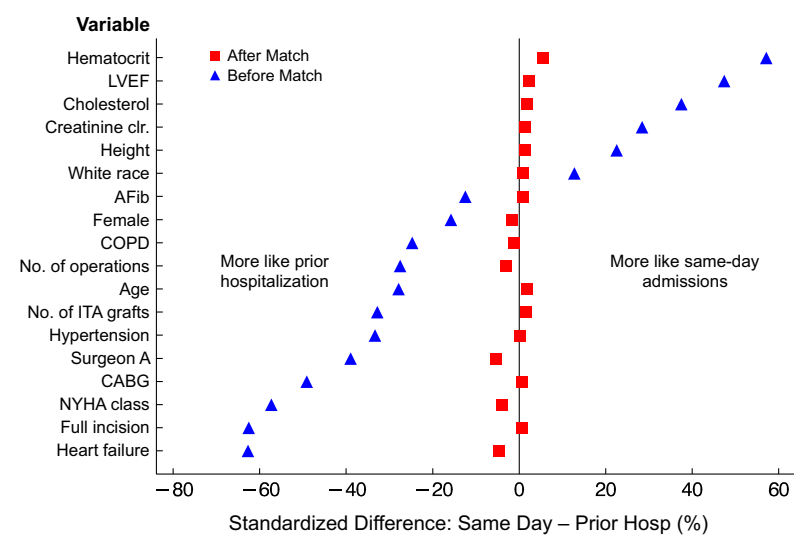

FIGURE 2. Covariance balance plot before and after propensity matching for patients in the same-day admission and prior hospitalization groups, standardized for comparability. ${ }^{24} L V E F$, Left ventricular ejection fraction; $C l r$, clearance; $A F i b$, atrial fibrillation; $C O P D$, chronic obstructive pulmonary disease; ITA, internal thoracic artery; $C A B G$, coronary artery bypass grafting; NYHA, New York Heart Association.

infections: 0 days, $5.73 \%$; 1 day, $11.86 \%$; $2-5$ days, $15.79 \%$; and $6-10$ days, $18.24 \%$.

Temporal trends. The percentages of patient transfers to our health care facility and those admitted preoperatively were relatively unchanged over the duration of this investigation. Infectious complications decreased over time, however, and never reached zero. We attributed this trend to several institution-specific factors: movement of cardiothoracic services to a new facility, implementation of and increased compliance efforts for several quality initiatives, such as more aggressive glucose control, hand washing, efforts to reduce central line-associated bloodstream infection, and more appropriate antibiotic stewardship. One would expect that implementation of these infection control initiatives over this time period would serve to decrease the prevalence of infections similarly for both groups.

Risk factors. Our reported findings of older age, obesity, diabetes, immunosuppressive therapy, emergency surgery, and red blood cell transfusion are similar to other reports on risk factors for postoperative infectious complications. ${ }^{1,3,16-18}$ Although identification of patient-specific risk factors for infection is important, many of these are unfortunately not modifiable at the time of surgical intervention.

\section{Mortality}

We found, like others, that once postoperative infections develop, they place patients at considerable risk of mortality and higher costs are incurred. ${ }^{4}$

\section{Clinical Implications}

Despite attempts to identify and implement initiatives to reduce postoperative infections, we are unable to eliminate them. Avoiding unnecessary preoperative admissions should be considered first, but it is difficult to determine the necessity of admission when multiple services are involved (medicine and surgery) in the care of these patients, and surgeons are often consulted days after hospitalization. Investigators have recommended research into strategies that could aid clinicians in deciding whether preoperative hospitalizations are necessary. ${ }^{6}$

Creating and assessing the cost, safety, and effectiveness of a new infection control strategy for hospitalized patients undergoing cardiac surgical procedures may serve to improve outcomes. For example, Huang and colleagues ${ }^{19}$ conducted a pragmatic randomized trial in adult patients in intensive care, examining targeted versus universal decolonization with daily chlorhexidine bathing to prevent infections. Universal decolonization reduced positive clinical cultures of methicillin-resistant Staphylococcus aureus by $37 \%$ and bloodstream infections from any pathogen by $44 \%$. Additional benefits noted by the investigators were a reduced environmental microbial burden and decreased risk for patient-to-patient transmission. More formalized policies in our own institution have been initiated, including

TABLE 2. Postoperative infectious complications by same-day admission and hospitalization before surgery: overall and matched groups

\begin{tabular}{|c|c|c|c|c|c|c|}
\hline \multirow[b]{3}{*}{ Complication } & \multirow{3}{*}{$\begin{array}{c}\begin{array}{c}\text { Total } \\
(\mathrm{n}=\mathbf{3 2 , 7 0 7 )} \\
\text { No. }(\%)\end{array}\end{array}$} & \multicolumn{2}{|c|}{ Same-day admission } & \multirow[b]{3}{*}{$\boldsymbol{P} *$} & \multicolumn{2}{|c|}{ Prior admission } \\
\hline & & $\begin{array}{c}\text { Before matching } \\
(\mathrm{n}=19,600) \\
\end{array}$ & $\begin{array}{l}\text { After matching } \\
(\mathrm{n}=\mathbf{7 8 1 4}) \\
\end{array}$ & & $\begin{array}{l}\text { After matching } \\
(\mathrm{n}=\mathbf{7 8 1 4}) \\
\end{array}$ & $\begin{array}{c}\text { Before matching } \\
(\mathrm{n}=13,107)\end{array}$ \\
\hline & & No. $(\%)$ & No. $(\%)$ & & No. $(\%)$ & No. $(\%)$ \\
\hline Postoperative composite infection & $2327(7.1)$ & $904(4.6)$ & $522(6.7)$ & $<.0001$ & $676(8.7)$ & $1423(11)$ \\
\hline \multicolumn{7}{|l|}{ Surgical site } \\
\hline Deep sternal wound & $317(0.97)$ & $163(0.83)$ & $99(1.3)$ & .7 & $93(1.2)$ & $154(1.2)$ \\
\hline Leg vein harvest site & $140(0.42)$ & $44(0.22)$ & $29(0.37)$ & .03 & $48(0.61)$ & $92(0.70)$ \\
\hline Arm vein harvest site & $5(0.02)$ & $3(0.015)$ & $2(0.025)$ & 6 & $1(0.013)$ & $2(0.015)$ \\
\hline Urinary tract infection & $986(3.0)$ & $403(2.1)$ & $225(2.5)$ & .02 & $278(3.6)$ & $583(4.4)$ \\
\hline Septicemia & $970(3.0)$ & $318(1.6)$ & $185(2.4)$ & $<.0001$ & $280(2.6)$ & $652(5.0)$ \\
\hline Pneumonia & $706(2.2)$ & $219(1.1)$ & $134(1.7)$ & .0001 & $203(2.6)$ & $487(3.7)$ \\
\hline
\end{tabular}

*Comparison of matched groups. 


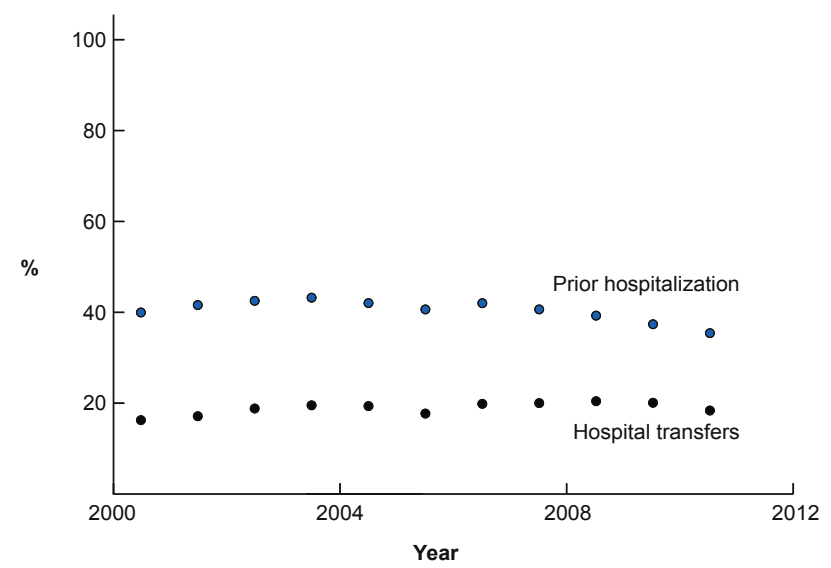

FIGURE 3. Percentage of patients hospitalized before cardiac surgery (blue). Approximately $20 \%$ of these were transfers from other health care facilities (black).

preoperative daily bathing and decolonization while awaiting operation.

\section{Limitations}

We are limited by the nature of our observational cohort study design whereby variables that are unaccounted for could influence our study findings. However, we included known pre- and intraoperative risk factors for postoperative infections and used modern statistical methods for risk adjustment. Our focus was on pre- and intraoperative variables that could lead to process improvement or modifiable risk factors. Hence, we did not include postoperative complications, which are hard to predict and may have occurred after the infectious complications.

Another important limitation of our study is the lack of data on the microbiology of infective organisms. Had these data been available, potential differences in patterns of infectious organisms between hospitalized

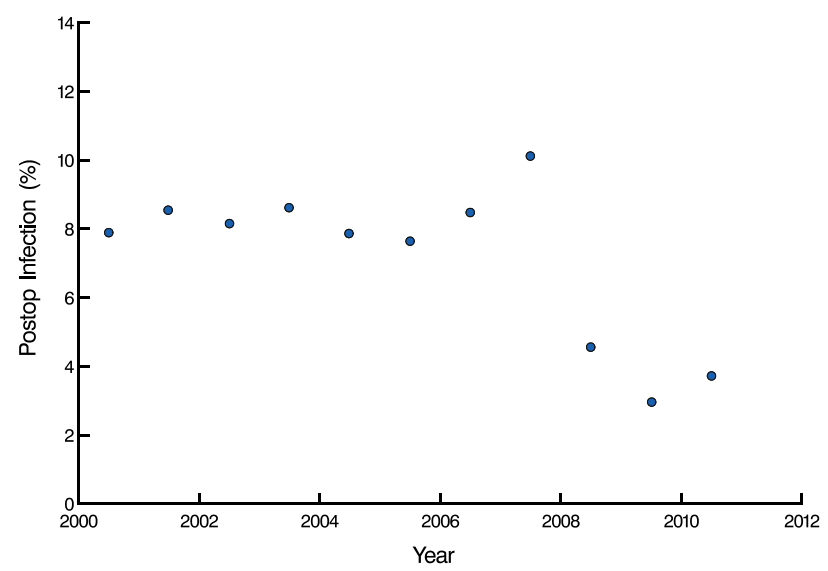

FIGURE 4. Percentage of patients developing a composite postoperative infectious complication in-hospital over time. Postop, Postoperative.

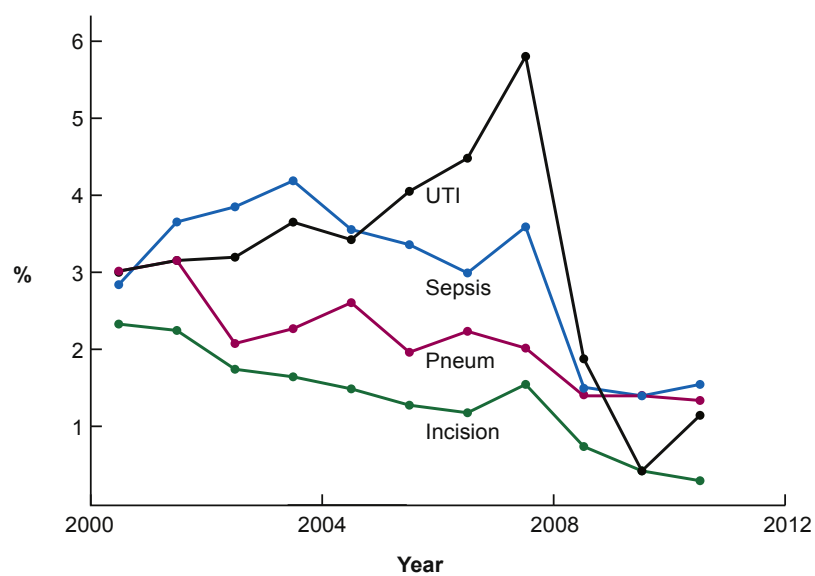

FIGURE 5. Percentage of patients developing specific postoperative infections over time. UTI, Urinary tract infection; Pneum, pneumonia.

patients and same-day admissions could have been identified, potentially leading to improvements in current infection control and prevention. Gardlund and colleagues ${ }^{20}$ reported that postoperative mediastinitis could be classified into groups based on microbiology and pathogenesis, for example: (1) mediastinitis associated with obesity, chronic obstructive pulmonary disease, and sternal dehiscence, typically caused by coagulase negative staphylococci; and (2) mediastinitis after perioperative contamination of the mediastinal space often caused by $S$ aureus.

A significant number of prior hospitalized patients were transferred from other hospitals. Specific data on hospitalization days at outside institutions were unavailable. Therefore, we were unable to examine the relationship between preoperative length of stay and subsequent infection sequelae. Similarly, data on the presence of invasive lines, urinary catheters, and ventilator dependency were not available, so their relationship with postoperative infections could not be examined.

We were unable to determine which patients truly needed inpatient hospitalization and which could have been stabilized and sent home to return as a same-day admission. Although we suggest universal decontamination of patients transferred from another hospital and those hospitalized before surgery, we were unable to determine with certainty whether this process improvement intervention would further reduce infectious complications.

We do not consider our use of a composite end point of infectious complications to be a limitation of our work. Our composite outcome data comprise complications of a similar nature and followed similar trends over time. Certainly, the use of composite end points combining dissimilar types of data should be approached with caution, as they are associated with several limitations. ${ }^{21-23}$ 
TABLE 3. Risk factors for postoperative infection (all, $\mathbf{n}=\mathbf{3 2 , 7 0 7}$ )

\begin{tabular}{|c|c|c|}
\hline Risk factor & Estimate $\pm \mathrm{SE}$ & $\boldsymbol{P}$ \\
\hline Prior hospitalization & $0.57 \pm 0.15$ & .0002 \\
\hline \multicolumn{3}{|l|}{ Demographics } \\
\hline Older age* & $0.24 \pm 0.043$ & $<.0001$ \\
\hline Female & $0.22 \pm 0.066$ & .0007 \\
\hline Weight $\dagger$ (heavier) & $0.30 \pm 0.04$ & $<.0001$ \\
\hline Height $\ddagger$ (shorter) & $-1.2 \pm 0.29$ & $<.0001$ \\
\hline Race: nonwhite & $0.15 \pm 0.069$ & .03 \\
\hline \multicolumn{3}{|l|}{ Status/symptoms } \\
\hline Emergency surgery & $0.74 \pm 0.12$ & $<.0001$ \\
\hline Higher NYHA class $\S$ & $0.022 \pm 0.0062$ & .0005 \\
\hline Immunosuppressant therapy & $0.24 \pm 0.057$ & $<.0001$ \\
\hline \multicolumn{3}{|l|}{ Comorbidities } \\
\hline Myocardial infarction & $0.13 \pm 0.052$ & .01 \\
\hline Chronic obstructive pulmonary disease & $0.35 \pm 0.053$ & $<.0001$ \\
\hline Prior stroke & $0.32 \pm 0.064$ & $<.0001$ \\
\hline Insulin-treated diabetes & $0.24 \pm 0.070$ & .0005 \\
\hline \multicolumn{3}{|l|}{ Laboratory values } \\
\hline BUN $\|$ & $0.30 \pm 0.079$ & .0002 \\
\hline Hematocrit $\Phi$ (lower) & $0.19 \pm 0.14$ & .17 \\
\hline Prior cardiac operation\# & $0.068 \pm 0.035$ & .05 \\
\hline \multicolumn{3}{|l|}{ Procedural } \\
\hline Aorta & $0.40 \pm 0.071$ & $<.0001$ \\
\hline Mitral valve surgery & $0.22 \pm 0.062$ & .0005 \\
\hline Coronary artery bypass grafting & $0.18 \pm 0.060$ & .003 \\
\hline Mitral valve replacement & $0.23 \pm 0.076$ & .003 \\
\hline Aortic valve replacement & $0.16 \pm 0.053$ & .003 \\
\hline Tricuspid valve surgery & $0.24 \pm 0.074$ & .001 \\
\hline Full incision & $0.22 \pm 0.10$ & .03 \\
\hline Intraoperative RBCs & $0.51 \pm 0.057$ & $<.0001$ \\
\hline Surgeon A & $0.29 \pm 0.081$ & .0004 \\
\hline Surgeon B & $0.29 \pm 0.14$ & .03 \\
\hline Earlier date of operation ${ }^{* *}$ & $-0.15 \pm 0.016$ & $<.0001$ \\
\hline More recent operation $\dagger \dagger$ & $0.28 \pm 0.049$ & $<.0001$ \\
\hline \multicolumn{3}{|l|}{ Risk factors unique to prior hospitalization } \\
\hline NYHA class II & $-0.31 \pm 0.070$ & $<.0001$ \\
\hline Lower LVEF & $-0.0070 \pm 0.0024$ & .004 \\
\hline Higher BUN & $0.0070 \pm 0.0026$ & .008 \\
\hline Higher bilirubin & $0.19 \pm 0.044$ & $<.0001$ \\
\hline \multicolumn{3}{|l|}{ Risk factors unique to same-day admission } \\
\hline Heart failure & $0.23 \pm 0.081$ & .005 \\
\hline Peripheral arterial disease & $0.33 \pm 0.10$ & .002 \\
\hline Treated diabetes & $0.22 \pm 0.086$ & .01 \\
\hline Surgeon C (less risk) & $-0.59 \pm 0.22$ & .008 \\
\hline Intercept & $-4.7 \pm 0.40$ & $<.0001$ \\
\hline
\end{tabular}

C-statistic $=.76 . S E$, Standard error; NYHA, New York Heart Association; $B U N$, blood urea nitrogen; $R B C$, red blood cells; $L V E F$, left ventricular ejection fraction. *(Age/ $50)^{2}$, squared transformation. $\dagger$ (Weight $\left.[\mathrm{kg}] / 80\right)^{2}$, squared transformation. $\ddagger($ Height $[\mathrm{cm}] / 180)^{2}$, squared transformation. $\S(\mathrm{NYHA} \text { class })^{2}$, squared transformation. |Logarithmic transformation. $\Phi(40 /$ hematocrit $)$, inverse transformation. \#Number of cardiac surgeries. ** Years from 1/1/2000. $\dagger \dagger$ Log transformation (years from 1/1/2000).

\section{CONCLUSIONS}

Despite the decrease in infectious complications after cardiac surgery over time, exposure to a health care facility before surgical intervention remains an important risk factor for infection and hospital mortality. When prior hospitalization is unavoidable, we empirically suggest daily universal decontamination for hospital transfers and patients hospitalized before surgery to further reduce postoperative infections. Additional investigation would provide more definitive information on whether the suggested process improvement initiative would further reduce infections.

\section{References}

1. Michalopoulos A, Geroulanos S, Rosmarakis ES, Falagas ME. Frequency, characteristics, and predictors of microbiologically documented nosocomial infections after cardiac surgery. Eur J Cardiothorac Surg. 2006;29:456-60.

2. Falagas ME, Rosmarakis ES, Rellos K, Michalopoulos A, Samonis G, Prapas SN. Microbiologically documented nosocomial infections after coronary artery bypass surgery without cardiopulmonary bypass. J Thorac Cardiovasc Surg. 2006;132:481-90.

3. Kollef MH, Sharpless L, Vlasnik J, Pasque C, Murphy D, Fraser VJ. The impact of nosocomial infections on patient outcomes following cardiac surgery. Chest. 1997;112:666-75.

4. Vogel TR, Dombrovskiy VY, Lowry SF. In-hospital delay of elective surgery for high volume procedures: the impact on infectious complications. J Am Coll Surg. 2010;211:784-90.

5. Bueno Cavanillas A, Rodriguez-Contreras R, Delgado Rodriguez M, Moreno Abril O, Lopez Gigosos R, Guillen Solvas J, et al. Preoperative stay as a risk factor for nosocomial infection. Eur J Epidemiol. 1991;7:670-6.

6. deFreitas DJ, Kasirajan K, Ricotta JJ 2nd, Veeraswamy RK, Corriere MA. Preoperative inpatient hospitalization and risk of perioperative infection following elective vascular procedures. Ann Vasc Surg. 2012;26:46-54.

7. Nagachinta T, Stephens M, Reitz B, Polk BF. Risk factors for surgical-wound infection following cardiac surgery. J Infect Dis. 1987;156:967-73.

8. Olsen MA, Krauss M, Agniel D, Schootman M, Gentry CN, Yan Y, et al. Mortality associated with bloodstream infection after coronary artery bypass surgery Clin Infect Dis. 2008;46:1537-46.

9. Shadish WR, Cook TD, Campbell DT. Experimental and Quasi-Experimental De signs for Generalized Causal Inference. Boston, Mass: Houghton Mifflin; 2002.

10. Rosenbaum PR, Rubin DB. The central role of the propensity score in observational studies for causal effects. Biometrika. 1983;70:41-55.

11. Blackstone EH. Comparing apples and oranges. J Thorac Cardiovasc Surg. 2002 $123: 8-15$.

12. Rubin DB. The design versus the analysis of observational studies for causal ef fects: parallels with the design of randomized trials. Stat Med. 2007;26:20-36.

13. Breiman L. Bagging predictors. Machine Learning. 1996;24:123-40.

14. Sauerbrei W, Schumacher M. A bootstrap resampling procedure for model building: application to the Cox regression model. Stat Med. 1992;11:2093-109.

15. Bergstralh EJ, Konsanke JL. Computerized Matching of Cases to Controls. Technical Report No. 56. Department of Health Science Research. Rochester, Minn: Mayo Clinic; 1995.

16. Lola I, Levidiotou S, Petrou A, Arnaoutoglou H, Apostolakis E, Papadopoulos GS. Are there independent predisposing factors for postoperative infections following open heart surgery? J Cardiothorac Surg. 2011;6:151.

17. Koch CG, Li L, Duncan AI, Mihaljevic T, Cosgrove DM, Loop FD, et al. Morbidity and mortality risk associated with red blood cell and blood-component transfusion in isolated coronary artery bypass grafting. Crit Care Med. 2006;34:1608-16.

18. Horvath KA, Acker MA, Chang H, Bagiella E, Smith PK, Iribarne A, et al. Blood transfusion and infection after cardiac surgery. Ann Thorac Surg. 2013;95:2194-201.

19. Huang SS, Septimus E, Kleinman K, Moody J, Hickok J, Avery TR, et al. Targeted versus universal decolonization to prevent ICU infection. $N$ Engl J Med. 2013;368:2255-65

20. Gardlund B, Bitkover CY, Vaage J. Postoperative mediastinitis in cardiac surgery microbiology and pathogenesis. Eur J Cardiothorac Surg. 2002;21:825-30.

21. Pocock SJ, Ariti CA, Collier TJ, Wang D. The win ratio: a new approach to the analysis of composite endpoints in clinical trials based on clinical priorities. Eur Heart J. 2012;33:176-82.

22. Tong BC, Huber JC, Ascheim DD, Puskas JD, Ferguson TB, Blackstone EH, et al. Weighting composite endpoints in clinical trials: essential evidence for the heart team. Ann Thorac Surg. 2012;94:1908-13.

23. Dmitrienko A, Wiens BL, Tamhane AC, Wang X. Tree-structured gatekeeping tests in clinical trials with hierarchically ordered multiple objectives. Stat Med. 2007; 26:2465-78.

24. Austin PC, Mamdani MM. A comparison of propensity score methods: a case-study estimating the effectiveness of post-MI statin use. Stat Med. 2006;25:2084-106. 
APPENDIX E1. Variables considered in the analyses

Patient

Demographics

Symptoms

Ventricular dysfunction

Cardiac comorbidity

Noncardiac comorbidity

Procedure
Age (y), * weight $(\mathrm{kg})$, height $(\mathrm{cm})$, weight/height ratio, body surface area $\left(\mathrm{m}^{2}\right),{ }^{*}$ body mass index $\left(\mathrm{kg} / \mathrm{m}^{2}\right),{ }^{*}$ gender, ${ }^{*}$ race* NYHA functional class (I-IV), ${ }^{*}$ NYHA functional class (I-IV) $<14 \mathrm{~d}$ before surgery, Canadian angina class, emergency operation

Previous myocardial infarction*

Atrial fibrillation, ${ }^{*}$ complete heart block or pacer,* ventricular arrhythmia, ${ }^{*}$ heart failure, ${ }^{*}$ endocarditis, ${ }^{*}$ prior cardiac surgery, $*$ total number of cardiac operations

Peripheral arterial disease, ${ }^{*}$ carotid disease, ${ }^{*}$ hypertension, ${ }^{*}$ pharmacologically treated diabetes, $*$ insulin-treated diabetes, non-insulin-treated diabetes, chronic obstructive pulmonary disease, ${ }^{*}$ history of smoking, ${ }^{*}$ preoperative renal dialysis, ${ }^{*}$ prior stroke, ${ }^{*}$ creatinine $(\mathrm{mg} / \mathrm{dL})$, ${ }^{*}$ blood urea nitrogen $(\mathrm{mg} / \mathrm{dL}),{ }^{*}$ bilirubin $(\mathrm{mg} / \mathrm{dL}),{ }^{*}$ creatinine clearance, ${ }^{*}$ glomerular filtration rate (Modification of Diet in Renal Disease), ${ }^{*}$ hematocrit (\%), * total cholesterol (mg/dL), high-density lipoprotein (mg/dL), low-density lipoprotein $(\mathrm{mg} / \mathrm{dL})$, triglycerides $(\mathrm{mg} / \mathrm{dL})$, red blood cell and component therapy transfusion Date of operation (years since January 1, 2000)*

NYHA, New York Heart Association. *Variables used in the propensity model.

Coronary artery bypass grafting, ${ }^{*}$ aortic valve procedure, ${ }^{*}$ mitral valve procedure, ${ }^{*}$ tricuspid valve procedure* 
TABLE E1. Baseline variables, perioperative factors, and mortality for unmatched patients by same-day admission and prior hospitalization status

\begin{tabular}{|c|c|c|c|c|c|}
\hline \multirow[b]{2}{*}{ Variable } & \multicolumn{2}{|c|}{ Same-day admission $(n=11,786)$} & \multicolumn{2}{|c|}{ Prior hospitalization $(n=5293)$} & \multirow[b]{2}{*}{$\boldsymbol{P}$} \\
\hline & $\mathbf{n}^{*}$ & No. $(\%)$ or mean \pm SD & n* & No. $(\%)$ or mean \pm SD & \\
\hline \multicolumn{6}{|l|}{ Demographics } \\
\hline Age (y) & 11,786 & $61 \pm 14$ & 5293 & $68 \pm 12$ & $<.0001$ \\
\hline Female & 11,786 & $3267(28)$ & 5293 & $2273(43)$ & $<.0001$ \\
\hline \multicolumn{6}{|l|}{ Race } \\
\hline White & 11,723 & $10,809(92)$ & 5213 & $4402(84)$ & $<.0001$ \\
\hline Black & 11,723 & $201(1.7)$ & 5213 & $656(13)$ & $<.0001$ \\
\hline Other & 11,723 & $713(6.1)$ & 5213 & $155(3.0)$ & $<.0001$ \\
\hline Body surface area $\left(\mathrm{m}^{2}\right)$ & 11,784 & $2.02 \pm 0.25$ & 5288 & $2.0 \pm 0.28$ & $<.0001$ \\
\hline Hospital transfer & 11,786 & $0(0)$ & 5259 & $2933(56)$ & - \\
\hline \multicolumn{6}{|l|}{ Clinical presentation } \\
\hline NYHA functional class & 11,783 & & 5273 & & $<.0001$ \\
\hline I & & $4298(36)$ & & $517(9.8)$ & \\
\hline II & & $6076(52)$ & & $1893(36)$ & \\
\hline III & & $1362(12)$ & & $1837(35)$ & \\
\hline IV & & $47(0.40)$ & & $1043(20)$ & \\
\hline Emergency surgery & 11,783 & $2(0.017)$ & 5292 & $358(6.8)$ & $<.0001$ \\
\hline \multicolumn{6}{|l|}{ Cardiac comorbidity } \\
\hline LVEF $(\%)$ & 9487 & $55 \pm 8.9$ & 4361 & $44 \pm 14$ & $<.0001$ \\
\hline Myocardial infarction & 11,786 & $1470(12)$ & 5293 & $3045(58)$ & $<.0001$ \\
\hline Heart failure & 11,786 & $933(7.9)$ & 5293 & $3285(62)$ & $<.0001$ \\
\hline Endocarditis & 11,786 & $328(2.8)$ & 5293 & $666(13)$ & $<.0001$ \\
\hline Atrial fibrillation/flutter & 10,601 & $721(6.8)$ & 5133 & 727 (14) & $<.0001$ \\
\hline Prior cardiac surgery & 11,785 & $1651(14)$ & 5293 & $1754(33)$ & $<.0001$ \\
\hline No. of cardiac operations & 11,786 & & 5293 & & $<.0001$ \\
\hline 1 & & $10,135(86)$ & & $3539(67)$ & \\
\hline 2 & & $1487(13)$ & & $1249(24)$ & \\
\hline$\geq 3$ & & $64(1.4)$ & & $505(9.5)$ & \\
\hline $\begin{array}{l}\text { Complete heart block or } \\
\text { pacer }\end{array}$ & 10,601 & $277(2.6)$ & 5133 & $365(7.1)$ & $<.0001$ \\
\hline Ventricular arrhythmia & 10,601 & $969(9.1)$ & 5133 & $493(9.6)$ & .4 \\
\hline \multicolumn{6}{|l|}{ Noncardiac comorbidity } \\
\hline Carotid disease & 11,786 & $1898(16)$ & 5293 & $2709(51)$ & $<.0001$ \\
\hline Hypertension & 11,786 & $6579(56)$ & 5293 & $4362(82)$ & $<.0001$ \\
\hline $\begin{array}{l}\text { Pharmacologically treated } \\
\text { diabetes }\end{array}$ & 11,724 & $1240(11)$ & 5159 & $1783(35)$ & $<.0001$ \\
\hline Insulin-treated diabetes & 11,690 & $370(3.2)$ & 5133 & $860(17)$ & $<.0001$ \\
\hline Peripheral arterial disease & 11,786 & $464(3.9)$ & 5293 & $1011(19)$ & $<.0001$ \\
\hline COPD & 11,786 & $992(8.4)$ & 5293 & $1373(26)$ & $<.0001$ \\
\hline Smoking & 11,720 & $5304(45)$ & 5323 & 3487 (67) & $<.0001$ \\
\hline Renal dialysis & 11,786 & $16(0.14)$ & 5293 & $254(4.8)$ & $<.0001$ \\
\hline Stroke & 11,786 & $310(2.6)$ & 5293 & $1060(20)$ & $<.0001$ \\
\hline Creatinine (mg/dL) & 11,760 & $1.01 \pm 0.36$ & 5270 & $1.46 \pm 1.32$ & $<.0001$ \\
\hline Bilirubin (g/dL) & 11,745 & $0.69 \pm 0.38$ & 5041 & $0.74 \pm 0.73$ & $<.0001$ \\
\hline $\begin{array}{l}\text { Glomerular filtration rate } \\
\text { (MDRD) }\end{array}$ & 11,760 & $80 \pm 20$ & 5288 & $67 \pm 33$ & $<.0001$ \\
\hline Hematocrit $(\%)$ & 11,785 & $40 \pm 4.8$ & 5291 & $34 \pm 6.0$ & $<.0001$ \\
\hline \multicolumn{6}{|l|}{ Procedure } \\
\hline Isolated CABG & 11,786 & $1532(13)$ & 5293 & $2033(38)$ & $<.0001$ \\
\hline Isolated valve & 11,786 & $6678(57)$ & 5293 & $1032(19)$ & $<.0001$ \\
\hline Combined CABG/valve & 11,786 & $1730(15)$ & 5293 & $1280(24)$ & $<.0001$ \\
\hline Aortic root, ascending aorta & 11,786 & 1477 (13) & 5293 & $790(15)$ & $<.0001$ \\
\hline Other cardiac & 11,786 & $369(3.1)$ & 5293 & $158(3.0)$ & .6 \\
\hline Surgical invasiveness & & & & & \\
\hline
\end{tabular}


TABLE E1. Continued

\begin{tabular}{|c|c|c|c|c|c|}
\hline \multirow[b]{2}{*}{ Variable } & \multicolumn{2}{|c|}{ Same-day admission $(n=11,786)$} & \multicolumn{2}{|c|}{ Prior hospitalization $(n=5293)$} & \multirow[b]{2}{*}{$\boldsymbol{P}$} \\
\hline & $\mathbf{n} *$ & No. $(\%)$ or mean \pm SD & $\mathbf{n}^{*}$ & No. $(\%)$ or mean \pm SD & \\
\hline Full incision & 11,786 & $7151(61)$ & 5293 & $5204(98)$ & $<.0001$ \\
\hline Less invasive & 11,786 & $4223(36)$ & 5293 & $67(1.3)$ & $<.0001$ \\
\hline Percutaneous/ports & 11,786 & $412(3.6)$ & 5293 & $22(0.41)$ & $<.0001$ \\
\hline $\mathrm{CPB}$ & 11,786 & $11,468(97)$ & 5293 & $4873(92)$ & $<.0001$ \\
\hline CPB time (min) & 11,786 & $89 \pm 38$ & 5293 & $113 \pm 58$ & $<.0001$ \\
\hline $\begin{array}{l}\text { Myocardial ischemic time } \\
(\min )\end{array}$ & 11,786 & $69 \pm 31$ & 5293 & $82 \pm 45$ & $<.0001$ \\
\hline Requirement for IABP & 11,786 & $63(0.53)$ & 5293 & $241(4.6)$ & $<.0001$ \\
\hline \multicolumn{6}{|l|}{ Blood product use } \\
\hline RBC transfusion & 11,784 & $3138(27)$ & 5288 & $4317(82)$ & $<.0001$ \\
\hline FFP & 11,784 & $689(5.8)$ & 5286 & $1597(30)$ & $<.0001$ \\
\hline Any blood product & 11,786 & $3501(30)$ & 5293 & $4408(83)$ & $<.0001$ \\
\hline $\begin{array}{l}\text { Immunosuppressive } \\
\text { therapy }\end{array}$ & 11,786 & $1620(14)$ & 5293 & 969 (18) & $<.0001$ \\
\hline \multicolumn{6}{|l|}{ Outcome } \\
\hline Postoperative infection & 11,786 & $383(3.2)$ & 5293 & 747 (14) & $<.0001$ \\
\hline In-hospital death & 11,786 & $47(0.4)$ & 5293 & $301(5.7)$ & $<.0001$ \\
\hline
\end{tabular}

